\title{
Verhaltenstherapie
}

\section{Band 17, Heft 2, Juni 2007}

\section{Editorial}

81 Eine Psychotherapie ist immer nur so gut wie ihre Diagnostik

Ehlert, U. (Zürich)

Originalarbeiten

84 Prospektiver Zusammenhang zwischen rigider und flexibler Kontrolle und Gewichtsverlust Timko, C.A. (Philalelphia, PA); Oelrich, C. (Hamburg); Lowe, M.R. (Philadelphia, PA)

90 Schlïsselstunden im Verlauf kognitiver Verhaltenstherapie von schizophrenen Patienten: Ein Beitrag zur Prozessforschung Kraemer, S. (München); Lihl, M. (Haar); Mergenthaler, E. (Ulm)

100 Stimmung und Therapieerfolg in der Psychotherapie Koban, C.; Willutzki, U. (Bochum)

109 Hilft wenig viel? Eine Minimalintervention für Patienten während der Wartezeit auf ambulante Verhaltenstherapie

Helbig, S.; Hoyer, J. (Dresden)

Übersichtsarbeit

116 Die Bedeutung von Metakognitionen für das Verständnis und die Psychotherapie von Zwang Neumann, A.; Geissner, E. (Prien am Chiemsee)

Für die Praxis

122 Die existenzielle Perspektive in der Verhaltenstherapie Noyon, A.; Heidenreich, T. (Frankfurt/M.)

Interview

129 Manfred Schedlowski: «Gezielte Verhaltensinterventionsprogramme können das biochemische Netzwerk im Körper beeinflussen» Schedlowski, M. (Essen)
Vol. 17, Issue 2, June 2007

Editorial

81 Successful Psychotherapy Always Needs Specific Diagnostic Procedures Ehlert, U. (Zürich)

Original Articles

84 Prospective Relationship of Rigid and Flexible Control and Weight Loss

Timko, C.A. (Philalelphia, PA); Oelrich, C. (Hamburg); Lowe, M.R. (Philadelphia, PA)

90 Key Sessions in the Course of Cognitive Behavioural Therapy with Schizophrenic Patients: A Contribution to Process Research Kraemer, S. (München); Lihl, M. (Haar); Mergenthaler, E. (Ulm)

100 Patients' Mood and Psychotherapeutic Outcome Koban, C.; Willutzki, U. (Bochum)

109 A Minimal Intervention for Waiting List Patients in Outpatient Behavior Therapy Helbig, S.; Hoyer, J. (Dresden)

Review Article

116 The Importance of Metacognitions in the Understanding and Treatment of Obsessive Compulsive Disorder Neumann, A.; Geissner, E. (Prien am Chiemsee)

For the Practitioner

122 Existential Perspective in Behaviour Therapy Noyon, A.; Heidenreich, T. (Frankfurt/M.)

Interview

129 Manfred Schedlowski: 'Tailored Behavioral Intervention Programs Are Required to Affect Physiological Responses and Disease Outcome' Schedlowski, M. (Essen)

\begin{tabular}{ll}
\hline KARGER & @ 2007 S. Karger GmbH, Freiburg \\
Fax +49 761 45207 14 & Artikel (Volltext) und Inhaltsverzeichnisse \\
$\begin{array}{l}\text { E-mail Information@Karger.de } \\
\text { www.karger.com }\end{array}$ & sowie das vorläufige Inhaltsverzeichnis des nächsten Heftes: \\
www.karger.com/ver_bk.htm
\end{tabular}




\section{Verhaltenstherapie}

\section{Band 17, Heft 2, Juni 2007}

132 Buchbesprechungen

137 Fort- und Weiterbildung

140 Mitteilungen der Verbände

142 Tagungen und Kongresse

128 Impressum

U3 Hinweise für Autoren (3. Umschlagseite)

U2 Erläuterungen zum Titelbild (2. Umschlagseite) Leib., C.; Vogt, B.; Izadi Yazdi, S. (Prien am Chiemsee)

Einen Ausblick auf den Inhalt der kommenden Hefte finden Sie auf Seite 144.

\section{Vol. 17, Issue 2, June 2007}

132 Book Reviews

137 Education

140 Information by Behavior Therapy Associations

142 Meetings and Conferences

128 Imprint

C3 Guidelines for Authors (Inside back cover)

C2 Explanations on the Title (Inside front cover) Leib., C.; Vogt, B.; Izadi Yazdi, S. (Prien am Chiemsee)

Forthcoming papers are listed on page 144. 